\title{
The methylation state of VDR gene in pulmonary tuberculosis patients
}

\author{
Chao Jiang ${ }^{1}$, Junjie Zhu ${ }^{1}$, Yuxiao Liu ${ }^{2}$, Xidi Luan ${ }^{2}$, Yan Jiang ${ }^{1}$, Gening Jiang ${ }^{1}$, Jiang Fan ${ }^{1}$ \\ ${ }^{1}$ Department of Thoracic Surgery, Shanghai Pulmonary Hospital Affiliated Tongji University, Shanghai 200433, China; ${ }^{2}$ Shandong Energy Zibo \\ Mining Group Co., LTD Central Hospital, Zibo 255120, China \\ Contributions: (I) Conception and design: C Jiang, J Zhu, G Jiang, J Fan; (II) Administrative support: G Jiang, J Fan; (III) Provision of study materials \\ or patients: All authors; (IV) Collection and assembly of data: C Jiang, J Zhu, Y Liu, X Luan, Y Jiang; (V) Data analysis and interpretation: All \\ authors; (VI) Manuscript writing: All authors; (VII) Final approval of manuscript: All authors. \\ Correspondence to: Dr. Jiang Fan. Department of Thoracic Surgery, Shanghai Pulmonary Hospital Affiliated Tongji University, 507 Zhengmin \\ Road, Shanghai 200433, China. Email: drjiangfan@yahoo.com; Dr. Gening Jiang. Department of Thoracic Surgery, Shanghai Pulmonary Hospital \\ Affiliated Tongji University, 507 Zhengmin Road, Shanghai 200433, China. Email: geningiiang@tongii.edu.cn.
}

Background: Our previous study suggested that the methylation of vitamin D receptor $(V D R)$ gene affected its expression and the defense against tuberculosis (TB) infection in vitro. In this study, we further investigated the methylation level of $V D R$ gene in pulmonary TB patients.

Methods: The consecutive TB patients who were admitted into our department from January 2013 to January 2015 were recruited. The potential methylation sites analyzed in this study included 16 CpG sites. The amplification of bisulfite modified genomic DNA was ligated to pUC18-T vector and the positive clone of blue-white selection was sequenced to analyze the methylation state of $V D R$ gene.

Results: This study had samples of $27 \mathrm{~TB}$ patients and 30 healthy controls. TB patients were in the hypermethylation state compared to the healthy controls. The significant differences between TB patients and healthy controls were detected in 7 of these $16 \mathrm{CpG}$ sites. The mRNA expression of AKT, GSK3 $\beta$ and FOXO1 decreased in TB patients compared to that of healthy controls.

Conclusions: Our study contributes to supplying more evidences that the methylation level of $V D R$ gene affects the progression of TB.

Keywords: Tuberculosis (TB); vitamin D receptor $(V D R)$ gene; methylation

Submitted Apr 15, 2017. Accepted for publication Sep 04, 2017.

doi: $10.21037 /$ jtd.2017.09.107

View this article at: http://dx.doi.org/10.21037/jtd.2017.09.107

\section{Introduction}

Tuberculosis (TB) is the second leading cause of death in infectious diseases around the world. According to "Global tuberculosis report 2015" published by WHO, there were 9.6 million new infections and 0.48 million multi-drug resistant (MDR) TB in 2014. Mycobacterium TB complex (MTBC) is the main pathogen of TB (1).

Any of the organisms in the MTBC, which includes Mycobacterium TB, Mycobacterium africanum, Mycobacterium bovis and Mycobacterium microti, can cause TB. Although the consistency on genome sequence among MTBC is over $99 \%$, each lineage is associated with different virulence. The virulence of different strains might be regulated by DNA methylation according to the whole-genome study (2). Furthermore, several genes which were associated with TB susceptibility, like NRAMP1 (3) and IFNG (4), have been subjected to epigenetic modification.

Epigenetics refers to the mechanism which result in gene expression changes without DNA sequence changes. The frequent modification includes DNA methylation and acetylation (5). In infectious diseases, DNA methylation in enhancers plays a critical role in regulating 
the transcriptional response to infection, even in nonproliferating cells (6). The pathogenic bacteria infected human dendritic cells (DCs) immediately caused the active demethylation at thousands of loci. Although more and more studies were reported, epigenetics of genes associated with TB susceptibility remain largely untapped field.

Vitamin D receptor (VDR) is a ligand-dependent transcription factor which mediated the activation of $1,25(\mathrm{OH})_{2} \mathrm{D}_{3}(7)$. It predominantly located in the nuclei of target cells. Upon the binding of vitamin D, VDR forms a heterodimer with retinoid $\mathrm{x}$-receptor (RXR). VDR complex regulates the activity of target molecules through binding to the specific vitamin D response elements (VDRE). $V D R$ gene is one of the key components in the host defense against TB $(8,9)$. The polymorphism of $V D R$ gene contributes to the risk of TB. Our previous study suggested that the methylation of $V D R$ gene affected its expression and the defense against TB infection in vivo (10). In this study, we further investigated the methylation level of $V D R$ gene in TB patients and analyzed the correlation between the methylation level of $V D R$ gene and patient phenotype. Our data confirmed the correlation between methylation of $V D R$ gene and the occurrence of TB.

\section{Methods}

\section{Ethical approval}

The protocol was approved by the Institutional Review Board (IRB) of Shanghai Pulmonary Hospital (Tongji University) (No. 2014-016). All participants signed the written informed consent.

\section{Patients and specimen collection}

The consecutive TB patients who were admitted into our department from January 2013 to January 2015 were recruited. The diagnosis of TB was based on the "Diagnostic criteria for tuberculosis (WS288-2008)" which was made by National Health and Family Planning Commission of the PRC and the Xpert MTB/RIF test which was recommended by the World Health Organization. Patients with chronic obstructive pulmonary diseases, asthma, lung cancer, diabetes and hypertension were excluded. The inclusive criteria of healthy controls included: negative on TB detection of chest $\mathrm{X}$-ray, peripheral blood and sputum; no consanguinity with TB patients; no history of chronic obstructive pulmonary diseases, asthma, lung cancer, diabetes and hypertension. The clinical and pathological data of participants which was recorded twice by two physicians respectively to ensure the accuracy were collected for analysis.

\section{Methylation state analysis}

The potential methylation sites analyzed in this study were "TTTTTGTGTTTTTTTTTTTATTTTCGTGTTTA TAGATCGTTTTGGGGTGTAGGACGTCGCGTTG ATTGAGGTTATTTAGGATCGTTTGTTTAATACA CTGCAGACGTACATCCGCTGCCGCCACCCGCCC CCGGGCAGCCACCTGCTCTATGCCAAGATGATC CAGAAGCTAGCCGACCTGCGCAGCCTCAATGAG GAGCACTCCAAGCAGTACCGCTGCCTCTCCTTC CAGCCTGAGTGCAGCATGAAGCTAACGCCCCTT GTGCTCG”. There were $16 \mathrm{CpG}$ sites in this sequence. The genomic DNA went through bisulfite modification with the CpGenome DNA Modification Kit (Intergen Company, Purchase, NY, USA). After PCR amplification, purification, the fragment was ligated to $\mathrm{pUC18}-\mathrm{T}$ vector. The positive clone of blue-white selection was sequenced.

\section{QPCR}

Total RNA was isolated for real-time PCR analysis to measure mRNA levels of the AKT, GSK $3 \beta$ and FOXO1 genes. Data shown are the relative abundance of the indicated mRNA normalized to that of GAPDH.

\section{Statistical analysis}

$\chi^{2}$ tests were performed to analyze the association between the methylation level of $V D R$ gene and other clinicopathological data. All data were analyzed through the SPSS package. P values $<0.05$ were considered statistically significant.

\section{Results}

\section{Clinicopathological characteristics}

During our investigation, 27 TB patients met the inclusive standards. All of them are Han people. The mean age of them was 41.36 years old. Of these patients, 18 (66.67\%) were male. Most patients had never smoked. Age- and gender-matched healthy controls (30 controls) were also recruited.

\section{The methylation state of VDR gene}

We analyzed the methylation state of $V D R$ gene through 
direct sequencing. The fragment (269 bp) of $V D R$ gene which contains $16 \mathrm{CpG}$ sites were sequenced. In general, TB patients were in the hyper-methylation state compared to the healthy controls. The significant differences between TB patients and healthy controls were detected in 7 of these $16 \mathrm{CpG}$ sites $(\mathrm{P}<0.05)$ (Figure 1).

\section{Correlation between methylation state of VDR gene and activity of AKT signal pathway}

Having established $V D R$ gene was in hyper-methylation level in TB patients, we next analyzed the correlation between methylation state of VDR gene and the mRNA expression of key components in AKT signaling pathway. The mRNA expression of $A K T, G S K 3 \beta$ and FOXO1 decreased in TB patients compared to that of healthy controls (Figure 2).

\section{Discussion}

The aberrant methylation was correlated with several diseases including autoimmunity diseases (11), diabetes,

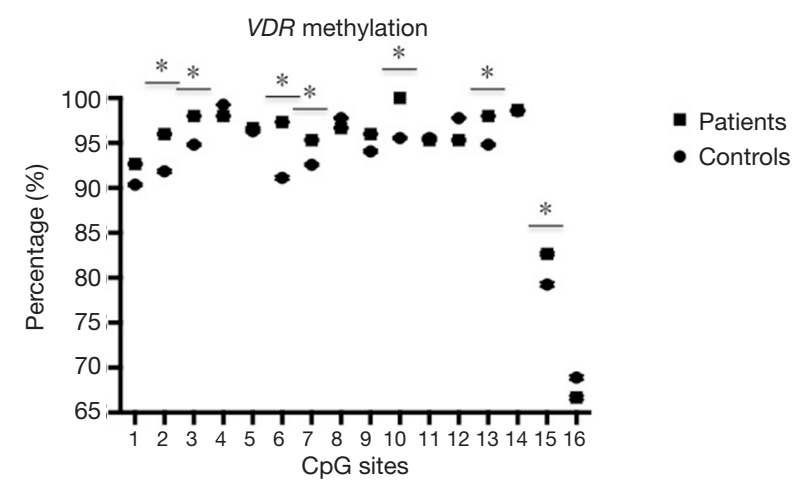

Figure 1 The methylation state of $V D R$ gene in TB patients and healthy controls. VDR, vitamin D receptor; TB, tuberculosis. and cancer. In infectious diseases, a growing number of studies have shown that DNA methylation plays a critical role in regulating the differentiation of immune cells and the expression of cytokines (12-15). The methylation level of several genes, like IL-4, IL-10, IFN- $\gamma$ and TNF- $\alpha$ had great changes with infection of bacterial and virus (16-18). In inflammatory bowel disease (IBD), the methylation state of IFNG might regulate the secretion of cytokines. The methylation level of lamina propria $T$ lymphocyte was significantly decreased in IBD patients, while the mRNA level of IFNG gene was increased 3 times (19). In this study, our data suggested that the $V D R$ gene were in hypermethylation in TB patients compared to healthy controls. The mRNA expression of key components in AKT signaling pathway decreased in TB patients. Combined with our previous study which suggested that the methylation changes of $V D R$ gene also affected the expression of IL$1 \beta$, IL- 6 and TNF- $\alpha$ (10), these studies contributed more evidences that the methylation level of $V D R$ gene might affect the progression of TB.

Currently, TB is diagnosed by chest $\mathrm{X}$-ray, direct staining or culture of the sputum samples and nucleic acid amplification techniques. However, all of them suffer from their own limitations. The image of chest X-ray usually confused with previous TB infection or inflammation of lung. DNA methylation state of $V D R$ genes can be directly detected in peripheral blood which provides an efficient method for diagnosis. This needs to be further studied in the future.

DNA methylation regulated the expression of target molecules through directly interfere the combination of transcription factors or binding with the methyl-CpG binding domain protein (MBD) (20). The expression not only changes in time, but also in location and manner. In TB, no evidence showed MBD was involved in the changes resulting from methylation modification of $V D R$. We assumed that $V D R$ might bind to corresponding
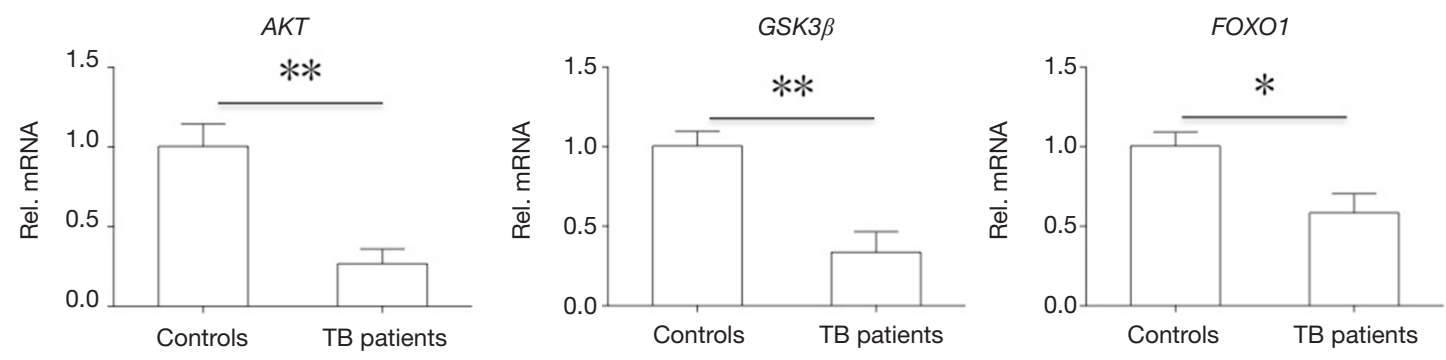

Figure 2 The mRNA expression level of $A K T$, GSK3 $\beta$ and FOXO1 gene. 
components directly to regulate the gene expression.

As DNA methylation is closely related to several diseases, it has the potential to be an ideal target for the treatment of these diseases. Furthermore, it already has been put into practice. Azacitidine, the first approved DNA methylation inhibitor by the US Food and Drug Administration (FDA), is experiencing phase III clinical trial for treatment of myelodysplastic syndrome. In $\mathrm{TB}$, the research has just begun. But it might contribute to provide a new solution in diagnosis and treatment.

\section{Acknowledgements}

We appreciate the patients and their families for participating in this study.

Funding: This work was supported by grants from the National Natural Scientific Foundation of China (81370107 and 81502383), the Natural Scientific Foundation of Science and Technology Commission of Shanghai Municipality (134119b1002), the Natural Scientific Foundation of Shanghai Municipal Commission of Health and Family Planning (20134279) and Scientific research project of Shanghai Municipal Commission of Health and Family Planning (2016Y0121; 2013ZYJB0401 and 2013sy023).

\section{Footnote}

Conflicts of Interest: The authors have no conflicts of interest to declare.

Ethical Statement: The protocol was approved by the Institutional Review Board (IRB) of Shanghai Pulmonary Hospital (Tongji University) (No. 2014-016). All participants signed the written informed consent.

\section{References}

1. Ueyama M, Chikamatsu K, Aono A, et al. Sub-speciation of Mycobacterium tuberculosis complex from tuberculosis patients in Japan. Tuberculosis (Edinb) 2014;94:15-9.

2. Zhu L, Zhong J, Jia X, et al. Precision methylome characterization of Mycobacterium tuberculosis complex (MTBC) using PacBio single-molecule real-time (SMRT) technology. Nucleic Acids Res 2016;44:730-43.

3. Zeng J, Xie L, Luo H, et al. The Epigenetic Modifications of Genes Associated with Tuberculosis Susceptibility and Implications for Epi-Drugs. Crit Rev Eukaryot Gene Expr
2015;25:349-62.

4. Aune TM, Collins PL, Collier SP, et al. Epigenetic Activation and Silencing of the Gene that Encodes IFN- $\gamma$. Front Immunol 2013;4:112.

5. Almouzni G, Cedar H. Maintenance of Epigenetic Information. Cold Spring Harb Perspect Biol 2016;8.

6. Pacis A, Tailleux L, Morin AM, et al. Bacterial infection remodels the DNA methylation landscape of human dendritic cells. Genome Res 2015;25:1801-11.

7. Carlberg C, Polly P. Gene regulation by vitamin D3. Crit Rev Eukaryot Gene Expr 1998;8:19-42.

8. Liu PT, Stenger S, Li H, et al. Toll-like receptor triggering of a vitamin D-mediated human antimicrobial response. Science 2006;311:1770-3.

9. Huang L, Liu C, Liao G, et al. Vitamin D Receptor Gene FokI Polymorphism Contributes to Increasing the Risk of Tuberculosis: An Update Meta-Analysis. Medicine (Baltimore) 2015;94:e2256.

10. Yang Y, Liu X, Yin W, et al. 5-Aza-2'-deoxycytidine enhances the antimicrobial response of vitamin D receptor against Mycobacterium tuberculosis. RSC Adv 2016;6:61740-6.

11. Ngalamika O, Liang G, Zhao M, et al. Peripheral whole blood FOXP3 TSDR methylation: a potential marker in severity assessment of autoimmune diseases and chronic infections. Immunol Invest 2015;44:126-36.

12. Morris AC, Spangler WE, Boss JM. Methylation of class II trans-activator promoter IV: a novel mechanism of MHC class II gene control. J Immunol 2000;164:4143-9.

13. Wright KL, Ting JP. Epigenetic regulation of MHC-II and CIITA genes. Trends Immunol 2006;27:405-12.

14. Wen H, Dou Y, Hogaboam CM, et al. Epigenetic regulation of dendritic cell-derived interleukin-12 facilitates immunosuppression after a severe innate immune response. Blood 2008;111:1797-804.

15. Fan XP, Zou ZQ, Long B, et al. Enhanced demethylation of interferon- $\gamma$ gene promoter in peripheral blood mononuclear cells is associated with acute-on-chronic hepatitis B liver failure. Tohoku J Exp Med 2011;224:13-9.

16. Gonsky R, Deem RL, Targan SR. Distinct Methylation of IFNG in the Gut. J Interferon Cytokine Res 2009;29:407-14.

17. Zhao M, Gao F, Wu X, et al. Abnormal DNA methylation in peripheral blood mononuclear cells from patients with vitiligo. Br J Dermatol 2010;163:736-42.

18. Fu LH, Ma CL, Cong B, et al. Hypomethylation of proximal $\mathrm{CpG}$ motif of interleukin-10 promoter regulates its expression in human rheumatoid arthritis. Acta 
Pharmacol Sin 2011;32:1373-80.

19. Kwon NH, Kim JS, Lee JY, et al. DNA methylation and the expression of IL-4 and IFN-gamma promoter genes in patients with bronchial asthma. J Clin Immunol 2008;28:139-46.

Cite this article as: Jiang $\mathrm{C}$, Zhu J, Liu Y, Luan X, Jiang Y, Jiang G, Fan J. The methylation state of $V D R$ gene in pulmonary tuberculosis patients. J Thorac Dis 2017;9(11):43534357. doi: 10.21037/jtd.2017.09.107
20. Moon MK, Lee YJ, Kim JS, et al. Effect of caffeic acid on tumor necrosis factor-alpha-induced vascular inflammation in human umbilical vein endothelial cells. Biol Pharm Bull 2009;32:1371-7. 